\title{
Caracterização dos estádios ontogenéticos de três espécies de palmeiras: uma proposta de padronização para estudos de dinâmica populacional ${ }^{1}$
}

\author{
RITA DE CÁSSIA QUITETE PORTELA ${ }^{2,4}$ e FLAVIO ANTONIO MAËS DOS SANTOS ${ }^{3}$
}

(recebido: 16 de março de 2011; aceito: 06 de outubro de 2011)

\begin{abstract}
Ontogenetic stages characterization for three palm species: a proposal of standardization for population dynamics studies). The objective of this study was to identify, classify and describe the ontogenetic stages and/or size classes of three tropical palm species, Astrocaryum aculeatissimum (Schott) Burret, Euterpe edulis Mart. and Geonoma schottiana Mart. The populations were sampled in five fragments of Atlantic Rain Forest in 2005. All individuals had their diameter and height measured, and also the number and kind of leaf recorded in each fragment of nine $30 \times 30 \mathrm{~m}$ plots. Based on these measurements, we assigned each individual to one of five classes. Astrocaryum aculeatissimum and E. edulis change their kind of leaf throughout development. Geonoma schottiana has a high variation in the kind of leaf, and only the seedlings stage displays only one kind of leaf. The appearance of stem and reproductive events also represents class change. The kind of leaf, the surge of the stem and reproductive events were the main characteristics used for the ontogenetic stage identification. However, for E. edulis and G. schottiana class identification, we also used quantitative characteristic because some stages have wide size variation. Studies that characterize and describe species ontogenetic stages are very important for standardization and to allow comparison between studies of population structure and dynamics.
\end{abstract}

Key words - Atlantic Rain Forest, Astrocaryum aculeatissimum, Euterpe edulis, Geonoma schottiana

RESUMO - (Caracterização dos estádios ontogenéticos de três espécies de palmeiras: uma proposta de padronização para estudos de dinâmica populacional). O objetivo desse estudo foi identificar, classificar e descrever os indivíduos de três espécies de palmeiras (Astrocaryum aculeatissimum (Schott) Burret, Euterpe edulis Mart. e Geonoma schottiana Mart.) em estádios ontogenéticos e/ou classes. As populações foram amostradas em 2005 em cinco fragmentos florestais. Todos os indivíduos das três espécies dentro de nove parcelas de $30 \times 30 \mathrm{~m}$ em cada fragmento foram medidos: diâmetro à altura do solo, altura até a inserção da última folha, número de folhas e partição do limbo foliar. Esses dados serviram para classificar os indivíduos de cada espécie em cinco classes. Astrocaryum aculeatissimum e E. edulis apresentaram marcada mudança no tipo de folha ao longo da ontogenia. Já G. schottiana apresentou ampla variação no tipo de folha e apenas as plântulas apresentaram um único tipo de folha. $\mathrm{O}$ aparecimento do estipe também marcou mudança de classe, assim como o evento reprodutivo. Tipo de folha, aparecimento do estipe e eventos reprodutivos foram as principais características usadas para identificação dos estágios ontogenéticos. Porém, para E. edulis e G. schottiana características quantitativas também foram utilizadas, pois alguns estádios apresentaram ampla variação no tamanho. Estudos que busquem a identificação e descrição de estádios ontogenéticos são muito importantes para possibilitar a padronização e comparação entre estudos populacionais.

Palavras-chave - Astrocaryum aculeatissimum, Euterpe edulis, Geonoma schottiana, Mata Atlântica

\section{Introdução}

Organismos passam por fases sequenciais do nascimento até a morte, que são geralmente caracterizadas pela idade cronológica. Essa sequência de fases de desenvolvimento de um indivíduo é chamada de ontogenia. Várias mudanças morfológicas, anatômicas,

1. Parte da tese do primeiro autor, Programa de Pós-Graduação em Ecologia, Universidade Estadual de Campinas, Caixa Postal 6109, 13083-970 Campinas, SP, Brasil.

2. Universidade Federal do Rio de Janeiro, Instituto de Biologia, Departamento de Ecologia, Caixa Postal 68020, 21941-590 Rio de Janeiro, RJ, Brasil.

3. Universidade Estadual de Campinas, Instituto de Biologia, Departamento de Biologia Vegetal, Caixa Postal 6109, 13083-970 Campinas, SP, Brasil.

4._Autor para correspondência: rita@quiteteportela.com.br fisiológicas e bioquímicas acontecem no curso da ontogenia (Gatsuk et al. 1980, Souza et al. 2000). Então, um dado indivíduo em um momento particular pode ser caracterizado não só pela sua idade cronológica mas, também, por critérios biológicos que indicam o seu estado de desenvolvimento (Gatsuk et al. 1980). E as características de mortalidade e natalidade dos indivíduos estão geralmente muito mais relacionadas com o estádio de desenvolvimento do que com a idade cronológica do mesmo. O termo "estado etário", criado por Gatsuk et al. (1980), refere-se à classificação dos indivíduos com base na ontogenia ou desenvolvimento da planta, como por exemplo, semente, plântula, juvenil e reprodutivo. De acordo com esses autores, o estado etário de um indivíduo pode ser definido por características quantitativas (por exemplo, altura) e qualitativas (por exemplo, tipo de 
folha). Os estados etários são geralmente caracterizados pelo aparecimento de estruturas que estavam ausentes em estádios mais recentes de desenvolvimento e pela perda de outras que estavam presentes. Portanto, o termo "estádio ontogenético" é o que tem sido mais usado para caracterizar as fases de vida das plantas e será aqui utilizado.

Estudos com o objetivo de descrição dos estádios ontogenéticos de plantas são escassos na literatura, mas muito importantes para a padronização na investigação de estrutura e dinâmica populacional de espécies de plantas. Souza et al. (2000) realizaram um estudo para a caracterização e comparação dos estádios pós-germinativos de Attalea humilis Mart. ex Spreng na região Norte do Estado do Rio de Janeiro e encontraram que indivíduos desta espécie em diferentes estádios apresentaram diferenças tanto no número de folhas quanto no tamanho, que tenderam a aumentar com o desenvolvimento ontogenético. Em um outro estudo, Bernacci et al. (2008) identificaram e descreveram os estádios ontogenéticos de uma população de Syagrus romanzoffiana (Cham.) Glassman em uma floresta estacional semidecídua em São Paulo. Os autores identificaram seis estádios, e estes apresentaram diferenças de tamanhos e de produção de folhas, embora alguns indivíduos de diferentes estádios tenham apresentado características semelhantes.

O objetivo deste estudo foi identificar e descrever os estádios ontogenéticos pós-germinativos das palmeiras Astrocaryum aculeatissimum (Schott) Burret, Euterpe edulis Mart. e Geonoma schottiana Mart. (Arecaceae), a partir de dados coletados em cinco populações naturais dessas espécies. Para tanto, admitimos que cada estádio ontogenético possa ser caracterizado por caracteres macromorfológicos externos e por certas propriedades (capacidade de produzir certas estruturas).

\section{Métodos e métodos}

Área de estudo - Cinco fragmentos florestais, com áreas de 3.500 ha (Reserva Biológica Poço das Antas, fragmento 1, 23K0778840 UTM7505080), 2.400 ha (Reserva Biológica União, fragmento 2, 23K0803350 UTM7515630), 57 ha (fragmento Santa Helena, fragmento 3, 23K0773000 UTM7506250), 21 ha (fragmento Estreito, fragmento 4, 23K0761800 UTM7496430) e 19 ha (fragmento Afetiva - Jorge, fragmento 5, 23K0760400 UTM7495000), foram escolhidos nos Municípios de Silva Jardim, Casimiro de Abreu e Rio das Ostras, na região Norte do Estado do Rio de Janeiro, dentro da Bacia Hidrográfica do Rio São João. Os fragmentos particulares estavam localizados num raio de até $30 \mathrm{~km}$ das Reservas.
Espécies estudadas - Foram escolhidas espécies de palmeiras nativas (Arecaceae), abundantes nas cinco áreas de estudo (Pires 2006).

Astrocaryum aculeatissimum é monóica, tolerante à sombra, de crescimento lento, de tronco simples ou múltiplo, formando touceira, com muitos espinhos (Henderson et al. 1995, Lorenzi et al. 2004). Atinge 4 a $8 \mathrm{~m}$ de altura e 11 a $15 \mathrm{~cm}$ de diâmetro. É uma espécie endêmica da Mata Atlântica, ocorrendo da Bahia a Santa Catarina (Henderson et al. 1995, Lorenzi et al. 2004). Apresenta reprodução sexuada e vegetativa. Possui alta produtividade de frutos (100 a 500 frutos/estipe). Os frutos são obovóides, com no máximo 3,0 a $3,5 \mathrm{~cm}$ de diâmetro (Henderson et al. 1995, Lorenzi et al. 2004) e são dispersados por roedores estocadores. A espécie é endêmica da Mata Atlântica do Brasil, ocupando o sub-bosque da floresta, bordas, clareiras e raramente o dossel quando reprodutivo, ocorrendo nas florestas de baixada em solos nunca inundados e ocasionalmente no pasto (Henderson et al. 1995). Ocorre em alta densidade (> 500 indivíduos ha $\left.{ }^{-1}\right)$ (Pires 2006).

Euterpe edulis (palmito juçara) é monóica, com tronco simples, tolerante à sombra e de crescimento lento. Atinge até $20 \mathrm{~m}$ de altura, ocupando o dossel da floresta, com 10 a $15 \mathrm{~cm}$ de diâmetro (Henderson et al. 1995, Lorenzi et al. 2004). Ocorre nas florestas costeiras, em encostas íngremes, e também em florestas mais interioranas, do nível do mar a $1.000 \mathrm{~m}$ de altitude (Henderson et al. 1995). Ocorre principalmente na Mata Atlântica costeira brasileira, mas sua distribuição atinge o interior até Brasília, Argentina e Paraguai (Henderson et al. 1995). Espécie que produz grande quantidade de frutos ( $>500$ frutos/estipe), dispersos por aves e mamíferos, se reproduz principalmente pela via sexual, habita o interior de florestas e ocorre naturalmente em alta densidade (100 a 500 indivíduos ha ${ }^{-1}$ ) em áreas bem preservadas (Pires 2006). Ocorre tipicamente em solos mais úmidos, muitas vezes em áreas brejosas (Silva-Matos 1995). A atividade de exploração do palmito, que causa o sacrifício da planta, está ocasionando o desaparecimento da espécie em algumas áreas (Henderson et al. 1995, Galetti \& Aleixo 1998).

Geonoma schottiana (ouricana) é monóica, de tronco simples, eventualmente formando touceira, tolerante à sombra e de crescimento lento. Atinge de 1 a $4 \mathrm{~m}$ de altura, ocupando o sub-bosque da floresta, e de 2,5 a $4 \mathrm{~cm}$ de diâmetro (Henderson et al. 1995, Lorenzi et al. 2004). Ocorre no Espírito Santo, Rio de Janeiro, São Paulo, Paraná, Santa Catarina, Minas Gerais e Goiás, na Mata Atlântica e no Cerrado (Henderson et al. 1995, Lorenzi et al. 2004). Espécies do gênero são restritas a áreas de floresta que recebem mais de $1.000 \mathrm{~mm}$ de chuva anual e possuem uma forte associação com o sub-bosque úmido e sombreado das florestas tropicais (Chazdon 1991). A espécie tem alta produtividade de frutos (100 a 500 frutos/estipe) dispersos por aves, reprodução sexual, habita o interior de florestas, bordas, clareiras e áreas abertas e ocorre em altas densidades (100 a 500 indivíduos ha ${ }^{-1}$ ) (Pires 2006). Na região estudada, as 
folhas são exploradas para a ornamentação de arranjos florais. Espécies de Geonoma são de difícil identificação (Chazdon 1991, Henderson 2011). Portanto, para a confirmação da identificação dessa espécie, foi levado material vegetativo e reprodutivo das cinco áreas de estudo para o taxonomista, Ricardo Reis, que está fazendo uma revisão do gênero e este confirmou se tratar de G. schottiana.

Desenho amostral - Em cada fragmento foram estabelecidas em abril e maio de 2005 nove parcelas de $30 \times 30 \mathrm{~m}$ (total: 0,81 ha) de forma sistemática. As parcelas foram distribuídas em três blocos, cada bloco com três parcelas distanciadas uma das outras por 50 metros e cada bloco com uma distância de $100 \mathrm{~m}$ entre si. Esse desenho foi adotado para que em todos os fragmentos fosse possível amostrar relativamente a mesma heterogeneidade ambiental. Um bloco foi estabelecido no meio do fragmento, e para cada direção no sentido Norte/Sul e Leste/Oeste outro bloco foi estabelecido. No caso das duas Reservas, ao invés de ter sido usado o meio do fragmento, foi escolhida uma trilha, por motivos práticos.

Dentro de cada fragmento, as áreas amostradas têm visualmente inclinação do terreno e umidade do solo similares para que sejam evitados efeitos diversos nas populações (Chazdon 1996, Scariot 1996), ou seja, foram evitadas áreas brejosas e áreas muito inclinadas.

Nessas parcelas foram amostrados todos os indivíduos das três espécies de palmeiras de junho a setembro de 2005.

Dados morfométricos e definição de estádios ontogenéticos Todos os indivíduos não reprodutivos nas parcelas, tiveram registrados o DAS (diâmetro a altura do solo - medido acima das raízes caulígenas aéreas na base do estipe - Alves et al. 2004), altura até a inserção da bainha da folha mais baixa (comprimento do estipe) e número de folhas. Para os indivíduos reprodutivos, foram registrados o DAS e altura. Apenas para os reprodutivos de G. schottiana foi contado o número de folhas. Cada indivíduo teve medido o DAS em duas direções para ser feita uma média (DAS médio). No caso de indivíduos sem estipe aparente (sem estipe acima do solo ou este coberto por bainhas foliares), não foi medida a altura. O DAS nesses casos representa o diâmetro das bainhas foliares, sendo dependente da quantidade de folhas produzidas pela planta, ou de bainhas que permaneceram aderidas ao caule.

Esses dados são importantes para auxiliar na distinção dos estádios ontogenéticos de cada espécie (Gatsuk et al. 1980), para consequente análise da estrutura e dinâmica das populações (Silva-Matos 1995, Souza et al. 2000) e para a padronização e comparação com estudos populacionais da mesma espécie. Outras características macro-morfológicas externas de cada planta foram observadas para a definição dos estádios ontogenéticos: partição do limbo foliar, podendo ser inteiro, pinatissecto ou transicional (parte inteira e parte pinatissecta), presença ou ausência de estipe aéreo e presença ou ausência de estruturas reprodutivas. Portanto, foram consideradas características qualitativas e quantitativas de cada planta. Para a caracterização das touceiras da espécie A. aculeatissimum foram cavadas 10 touceiras em 24 e 25 de outubro de 2006, sendo sete no fragmento Santa Helena e três na Reserva Biológica Poço das Antas. Cada clone ("indivíduo") da touceira foi classificado quanto ao seu estádio ontogenético, assim como os indivíduos solitários.

Todas as análises e gráficos foram realizadas no SYSTAT software 11 (SYSTAT 2004).

\section{Resultados}

Foram amostrados em 2005, 2.758 indivíduos de Astrocaryum aculeatissimum, 1.146 indivíduos de Euterpe edulis e 2.470 indivíduos de Geonoma schottiana, num total de 6.374 indivíduos das três espécies nos cinco fragmentos estudados. O número de indivíduos de cada espécie variou entre os fragmentos (tabela 1). A. aculeatissimum foi mais abundante num dos maiores fragmentos e menos abundante num dos menores. E. edulis e $G$. schottiana foram mais abundantes no maior fragmento e menos abundantes no segundo maior fragmento.

Dados morfométricos e definição de estádios ontogenéticos - Foram identificados cinco estádios ontogenéticos para $A$. aculeatissimum, quatro estádios pré-reprodutivos e um reprodutivo. Esses estádios foram definidos por características como ausência (três

Tabela 1. Número total de indivíduos por 1 ha amostrados em 2005 nos cinco fragmentos de Mata Atlântica localizados no Norte do Estado do Rio de Janeiro. (Fragmento 1. Reserva Biológica Poço das Antas, 3.500 ha; Fragmento 2. Reserva Biológica União, 2.400 ha; Fragmento 3. Santa Helena, 57 ha; Fragmento 4. Estreito, 21 ha; Fragmento 5. Afetiva-Jorge, 19 ha).

Table 1. Total number of individuals in 1 ha in the year of 2005 in five forest fragments in the Atlantic Rain Forest in the north of Rio de Janeiro State. (Fragment 1. Reserva Biológica Poço das Antas, 3.500 ha; Fragment 2. Reserva Biológica União, 2.400 ha; Fragment 3. Santa Helena, 57 ha; Fragment 4. Estreito, 21 ha, Fragment 5. Afetiva-Jorge, 19 ha).

\begin{tabular}{ccrc}
\hline Fragmento & $\begin{array}{c}\text { Astrocaryum } \\
\text { aculeatissimum }\end{array}$ & $\begin{array}{c}\text { Euterpe } \\
\text { edulis }\end{array}$ & $\begin{array}{c}\text { Geonoma } \\
\text { schottiana }\end{array}$ \\
\hline 1 & 381 & 1060 & 2255 \\
2 & 1547 & 17 & 7 \\
3 & 686 & 192 & 259 \\
4 & 52 & 33 & 459 \\
5 & (uma única touceira) & & \\
5 & 738 & 111 & 68 \\
\hline
\end{tabular}


estádios) e presença (dois estádios) de estipe aéreo. Os três estádios sem estipe aéreo foram definidos de acordo com o tipo de folha (figuras 1-9, tabela 2): plântula (figuras 1-2) - indivíduo com folhas bífidas; infante (figuras 3-4)-indivíduo com presença de folhas transicionais (transicional, entre bífida e pinada); jovem (figura 5) - indivíduo com folhas pinadas, sem presença de estipe aéreo. Os dois estádios com estipe aéreo foram definidos de acordo com a ausência ou presença de estruturas reprodutivas: imaturo ou virgem (figura 6) - indivíduo com estipe aparente, mas sem sinal de evento reprodutivo; reprodutivo ou adulto (figura 7) - indivíduo com sinal de evento reprodutivo (presença de inflorescência ou infrutescência). Essa espécie ainda ocorreu formando touceiras e cada indivíduo da touceira foi classificado de acordo com o estádio.

Plantas de A. aculeatissimum em diferentes estádios apresentaram diferenças marcantes no número de folhas $(H=805,905, g l=5, P<0,001)$, com aumento no número de folhas com o desenvolvimento ontogenético (figura 10). O DAS e a altura (figura 10) foram menores no estádio imaturo do que no reprodutivo $(H=22,081$, $g l=1, P<0,001)$, indicando que o tamanho, além da ausência de estruturas reprodutivas, é um indicador desses estádios.

Foram amostrados 89 rametas nas 10 touceiras cavadas de $A$. aculeatissimum. $\mathrm{O}$ número de rametas variou de 2 a 35 por touceira. Foram amostradas rametas em todos os estádios ontogenéticos nas 10 touceiras. Estipes sem folhas, encontrados nas touceiras, foram considerados como rametas mortas. Apenas em quatro touceiras foram encontrados indivíduos não clonais. Possivelmente esses são indivíduos estabelecidos dentro da touceira pela germinação de frutos produzidos na própria touceira. Ou seja, em $60 \%$ das touceiras cavadas, todos os "indivíduos" eram clonais (rametas), tendo o número de clones por touceira variado de 4 a 35 . Nas quatro touceiras $(40 \%)$ em que ocorreram indivíduos não clonais, sete deles não eram clonais (estabelecidos a partir de semente), variando de 1 a 3 por touceira, e quatro eram clonais, variando de 0 a 3 clones por touceira.

Tabela 2. Classificação e caracterização dos estádios ontogenéticos e classes estabelecidos para as espécies Astrocaryum aculeatissimum, Euterpe edulis e Geonoma schottiana em cinco fragmentos florestais de Mata Atlântica no Norte do Estado do Rio de Janeiro.

Tabel 2. Ontogenetic stages and classes classification and characterization of Astrocaryum aculeatissimum, Euterpe edulis e Geonoma schottiana in five fragments in the Atlantic Rain Forest in the north of Rio de Janeiro State.

\begin{tabular}{|c|c|c|c|c|}
\hline Estádio & $\begin{array}{l}\text { Tamanho } \\
\text { (DAS) }\end{array}$ & Estipe & $\begin{array}{c}\text { Estruturas } \\
\text { reprodutivas }\end{array}$ & $\begin{array}{l}\text { Tipo de } \\
\text { folhas }\end{array}$ \\
\hline \multicolumn{5}{|c|}{ Astrocaryum aculeatissimum } \\
\hline Plântula & & ausente & ausentes & somente bífida \\
\hline Infante & & ausente & ausentes & bífida e/ou transicional \\
\hline Jovem & & ausente & ausentes & pinada \\
\hline Imaturo & & presente & ausentes & pinada \\
\hline Reprodutivo & & presente & presentes & pinada \\
\hline \multicolumn{5}{|c|}{ Euterpe edulis } \\
\hline Plântula & $<52 \mathrm{~mm}$ & ausente & ausentes & somente leque \\
\hline Infante & $>52 \mathrm{~mm}$ & ausente & ausentes & pinada \\
\hline Jovem & & presente & ausentes & pinada \\
\hline Imaturo & & presente & ausentes & pinada \\
\hline Reprodutivo & & presente & presentes & pinada \\
\hline \multicolumn{5}{|c|}{ Geonoma schottiana } \\
\hline Plântula & & ausente & ausentes & somente bífida \\
\hline Infante & & ausente & ausentes & bífida, transicional ou pinada \\
\hline Jovem & & presente & ausentes & transicional ou pinada \\
\hline Imaturo & $<30 \mathrm{~mm}$ & presente & ausentes & transicional ou pinada \\
\hline Reprodutivo & $>30 \mathrm{~mm}$ & presente & presentes & transicional ou pinada \\
\hline
\end{tabular}



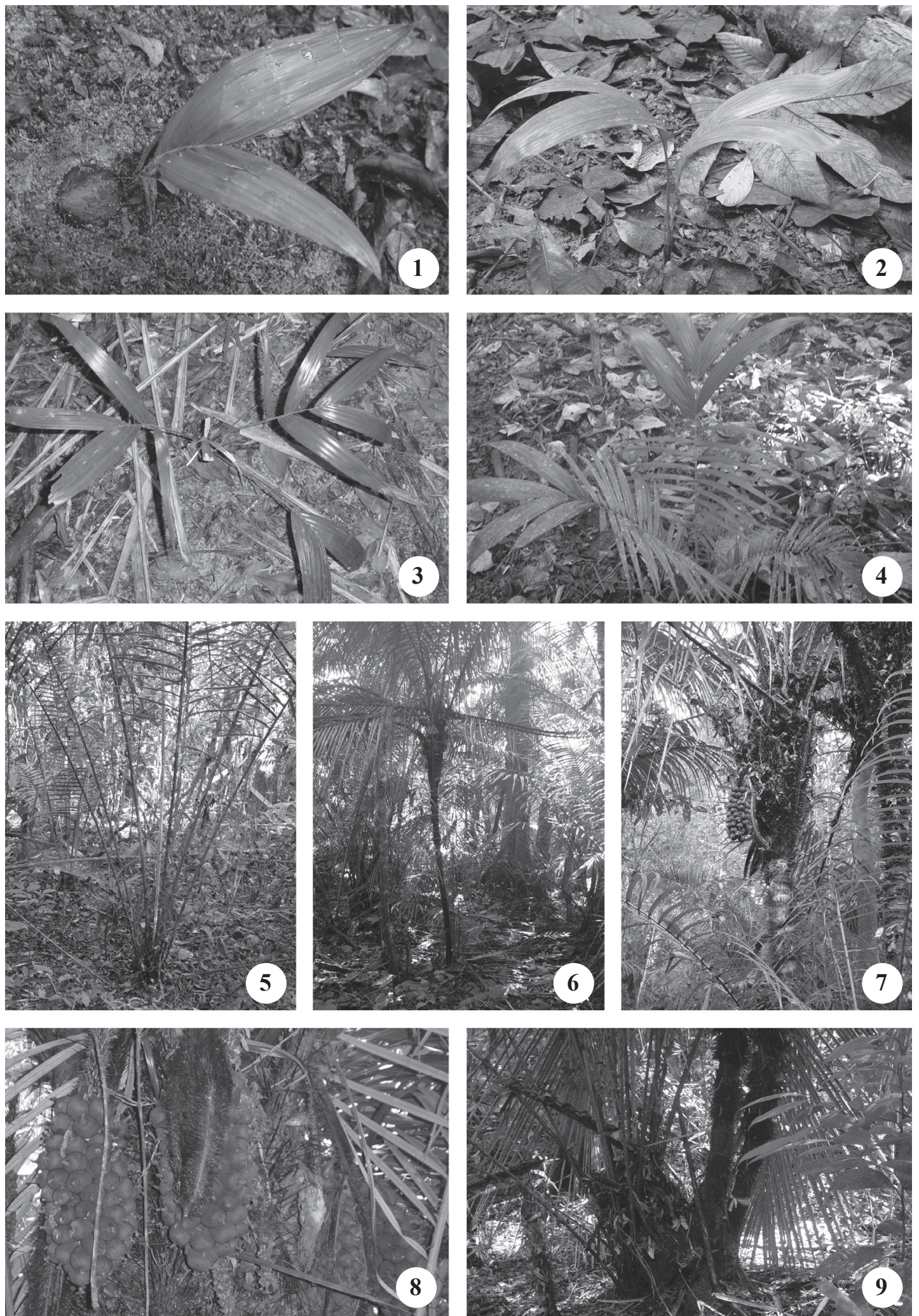

Figuras 1-9. Estádios ontogenéticos de Astrocaryum aculeatissimum nas áreas de estudo. 1-2. Plântula. 3-4. Infante. 5. Jovem. 6. Imaturo. 7. Reprodutivo. 8. Frutos. 9. Touceira. Mediana da altura dos indivíduos reprodutivos $=350 \mathrm{~cm}$.

Figures 1-9. Ontogenetic stages of Astrocaryum aculeatissimum in the studied areas. 1-2. Seedling. 3-4. Infant. 5. Juvenile. 6. Immature. 7. Reproductive. 8. Fruits. 9. Multi-stemmed form. Height median of reproductive adults $=350 \mathrm{~cm}$. 

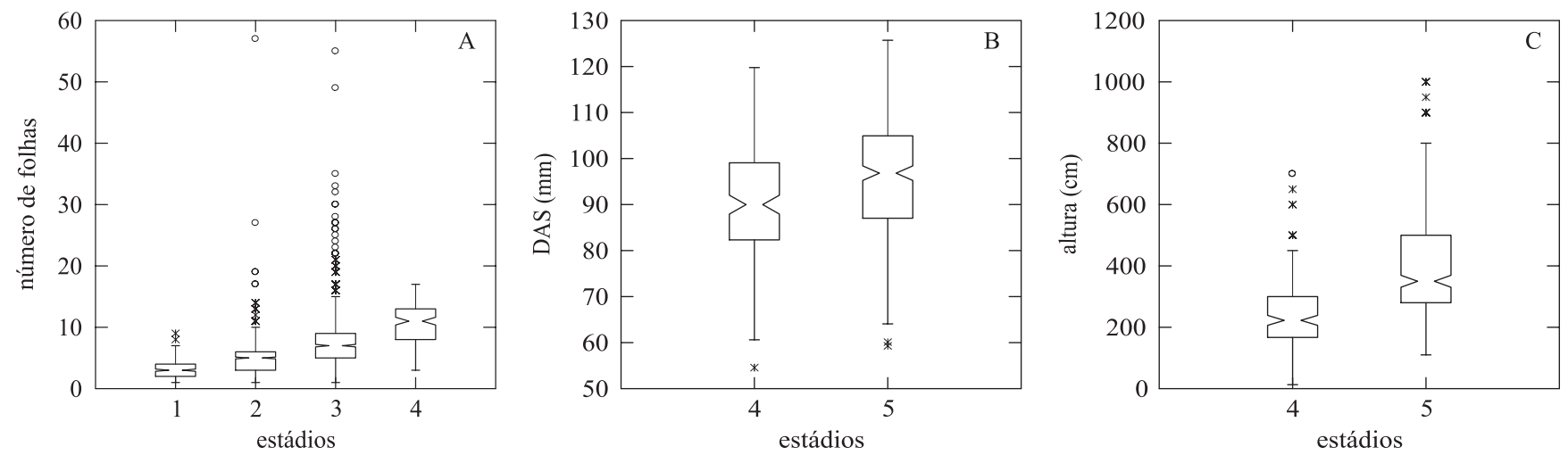

Figura 10. Número de folhas (A), DAS (B) e altura (C) de Astrocaryum aculeatissimum dos estádios ontogenéticos no Norte do Estado do Rio de Janeiro. 1. Plântula ( $n=295) ; 2$. Infante $(n=1.035) ; 3$. Jovem $(n=942) ; 4$. Imaturo $(n=167) ; 5$. Reprodutivo $(n=317)$. Cada caixa (box) representa 50\% das observações, as barras verticais representam a amplitude total de distribuição; * = valores extremos (outlier); $\mathrm{O}=$ os valores muito extremos. A porção "acinturada" da caixa representa o intervalo de confiança de $95 \%$ ao redor da mediana. Porções "acinturadas" que não se sobrepõem indicam diferenças significativas no nível de $95 \%$ de confiança.

Figure 10. Number of leaves (A), DAS (B) and height (C) of Astrocaryum aculeatissimum ontogenetic stages in the north of Rio de Janeiro State. 1. Seedling $(n=295) ; 2$. Infant $(n=1.035) ; 3$. Juvenile $(n=942) ; 4$. Immature $(n=167) ; 5$. Reproductive $(n=317)$. Each box represents $50 \%$ of the observation, the bars represent the amplitude of the observation; notches surrounding the median represent median $95 \%$ confidence intervals; $*=$ outlier; $O=$ extreme value. Non-overlapping of notches indicates significant difference at $95 \%$ confidence level.

Astrocaryum aculeatissimum se apresentou como indivíduos solitários e como touceira. Do total de 2.754 indivíduos amostrados em 2005 nos 5 fragmentos, mais da metade (56,6\%-1.558 indivíduos) ocorreram como touceira (tabela 3). Foram amostradas 306 touceiras, sendo que o número de rametas (clones) por touceira variou de 2 a 42, com uma mediana de 4 .

O número de indivíduos por touceira diferiu entre os fragmentos $(H=24,274, \mathrm{gl}=4, P<0,001)$. As touceiras no menor fragmento apresentaram um maior número de rametas do que nos demais fragmentos (figura 11).

Foram reconhecidos quatro estádios ontogenéticos em E. edulis, sendo três estádios pré-reprodutivos e um reprodutivo. Esses estádios foram definidos por características como ausência (dois estádios) e presença (dois estádios) de estipe aéreo. Os dois estádios sem estipe aéreo foram definidos de acordo com o aparecimento da folha madura (pinada). Os dois estádios com presença de estipe aéreo foram definidos de acordo com a ausência ou presença de estruturas reprodutivas. O estádio com presença de estipe aéreo, mas sem sinal de evento reprodutivo, foi dividido em duas classes de tamanho de acordo com o DAS. Para tanto, foi usada uma característica quantitativa (DAS) da definição de jovem elaborada por Carvalho et al. (1999). De acordo com essa característica, os autores classificaram como jovens aqueles indivíduos com estipe
Tabela 3. Número de indivíduos de todos os estádios ontogenéticos amostrados de Astrocaryum aculeatissimum e de indivíduos em touceiras, seguindo pela porcentagem, na coleta de dados de 2005 nos cinco fragmentos de Mata Atlântica localizados no Norte do Estado do Rio de Janeiro. Área amostrada por fragmento: 0,81 ha. (Fragmento 1. Reserva Biológica Poço das Antas, 3.500 ha; Fragmento 2. Reserva Biológica União, 2.400 ha; Fragmento 3. Santa Helena, $57 \mathrm{ha}$; Fragmento 4. Estreito, 21 ha; Fragmento 5. Afetiva-Jorge, 19 ha).

Tabel 3. Single-stemmed and multi-stemmed number of individuals of all ontogenetic stages of Astrocaryum aculeatissimum in 2005 in five forest fragments in the Atlantic Rain Forest in the north of Rio de Janeiro State. Total censused area in each fragment: 0.81 ha. (Fragment 1. Reserva Biológica Poço das Antas, 3,500 ha; Fragment 2. Reserva Biológica União, 2,400 ha; Fragment 3. Santa Helena, 57 ha; Fragment 4. Estreito, 21 ha; Fragment 5. Afetiva-Jorge, $19 \mathrm{ha}$ ).

\begin{tabular}{cccc}
\hline Fragmento & $\begin{array}{c}\text { Número de } \\
\text { indivíduos }\end{array}$ & $\begin{array}{c}\text { Número de indivíduos } \\
\text { em touceira }\end{array}$ & $\begin{array}{c}\text { Número de } \\
\text { touceiras }\end{array}$ \\
\hline 1 & 309 & $58(18,77 \%)$ & 14 \\
2 & 1253 & $633(50,51 \%)$ & 151 \\
3 & 552 & $322(58,33)$ & 65 \\
4 & 42 & $42(100 \%)$ & 1 \\
5 & 598 & $503(84,11 \%)$ & 75 \\
\hline
\end{tabular}




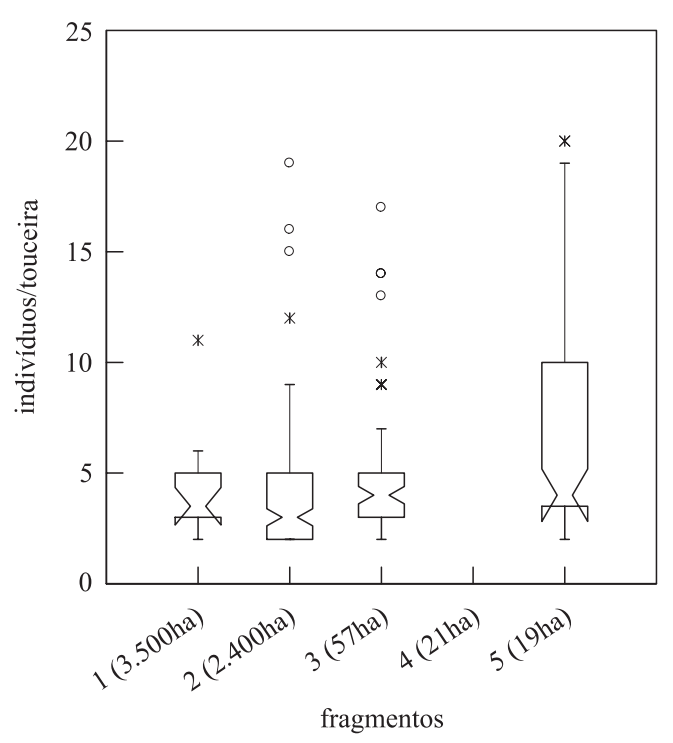

Figura 11. Número de indivíduos por touceira de Astrocaryum aculeatissimum em todos os estádios ontogenéticos em cinco fragmentos florestais de diferentes tamanhos (1. Reserva Biológica de Poço das Antas; 2. Reserva Biológica União; 3. Santa Helena; 4. Estreito (42 indivíduos em uma única touceira - dado excluído para facilitar a visualização); 5. Afetiva-Jorge no Norte do Estado do Rio de Janeiro na coleta de 2005). Cada caixa (box) representa 50\% das observações, as barras verticais representam a amplitude total de distribuição; * = valores extremos (outlier); O = valores muito extremos. A porção "acinturada" da caixa representa o intervalo de confiança de 95\% ao redor da mediana. Porções "acinturadas" que não se sobrepõem indicam diferenças significativas no nível de 95\% de confiança.

Figure 11. All ontogenetic stages number of individuals per multi-stemmed group of Astrocaryum aculeatissimum in five forest fragment of different sizes. (1. Reserva Biológica de Poço das Antas; 2. Reserva Biológica União; 3. Santa Helena; 4. Estreito (42 individuals in one multi-stemmed group this data was excluded to improve the visualization); 5. Afetiva-Jorge in the north of Rio de Janeiro State in 2005). Each box represents $50 \%$ of the observation, the bars represent the amplitude of the observation; notches surrounding the median represent median 95\% confidence intervals; * $=$ outlier; $\mathrm{O}=$ extreme value. Non-overlapping of notches indicates significant difference at 95\% confidence level.

aparente e com até $52 \mathrm{~mm}$ de DAS. Essa medida foi usada para permitir comparação e assim dar início a uma padronização para as classes da espécie. Portanto, para a definição das cinco classes, foram usadas tanto características quantitativas (DAS) como qualitativas (tipo de folha e ausência ou presença de estipe e sinal de evento reprodutivo), sendo (figuras 12-18, tabela 2): plântula (figuras 12-13) - indivíduo com folhas palmadas ou leque; infante (figuras 14-15) - indivíduo com a presença de folhas pinadas e sem estipe aparente; jovem (figura 16) - indivíduo com estipe aparente e DAS máximo de $52 \mathrm{~mm}$; imaturo ou virgem (figura 17) - indivíduo com estipe aparente, com DAS maior do que $52 \mathrm{~mm}$, mas sem sinal de evento reprodutivo; reprodutivo ou adulto (figura 18) - indivíduo com sinal de evento reprodutivo (presença de inflorescência ou infrutescência). Essa espécie não se apresentou em forma de touceiras, porém foram encontrados alguns indivíduos apresentando ramificação ao longo do estipe (Portela et al. 2009). Para a caracterização dos estádios, esses indivíduos foram retirados da análise por não serem característicos da população.

Plantas de E. edulis em diferentes estádios apresentaram diferenças quanto ao número de folhas ( $H=397,508, g l=2, P<0,001)$, com um aumento progressivo no número de folhas com o aumento do desenvolvimento ontogenético (figura 19). O mesmo aconteceu com o DAS $(H=985,131 \mathrm{gl}=4, P<0,001)$ e a altura $(H=610,235, g l=3, P<0,001)$ (figura 19), indicando que o estádio imaturo difere do reprodutivo não somente pela ausência de estrutura reprodutiva, como também pelo tamanho.

Foram reconhecidos três estádios ontogenéticos em G. schottiana, dois estádios pré-reprodutivos e um reprodutivo. Esses estádios foram definidos pela ausência (um estádio) ou presença (dois estádios) de estipe aéreo. O estádio sem estipe aéreo foi dividido em duas classes de tamanho de acordo com a presença ou ausência de DAS. Vale lembrar que nesses casos o DAS representa o diâmetro das bainhas foliares, sendo dependente da quantidade de folhas produzidas pela planta, ou de bainhas que permaneceram aderidas ao caule. Os dois estádios com presença de estipe aéreo foram definidos de acordo com a ausência ou presença de estruturas reprodutivas. O estádio com presença de estipe aéreo, mas sem sinal de evento reprodutivo, foi dividido em duas classes de tamanho de acordo com o DAS. Para tanto, o menor DAS apresentado por um indivíduo com sinal de evento reprodutivo foi usado como base para dividir essas duas classes. Portanto, para a definição das cinco classes, foram usadas tanto características quantitativas (DAS) como qualitativas (ausência ou presença de estipe e sinal de evento reprodutivo), sendo (figuras 20-26, tabela 2): plântula (figura 20) - indivíduo somente com folhas bífidas, sem DAS e sem estipe aparente; infante (figuras 21-22) - indivíduo com folhas bífidas, transicionais ou pinadas, com DAS formado pela bainha das folhas, mas sem estipe aparente; jovem (figura 23) - indivíduo com presença de estipe aéreo e 

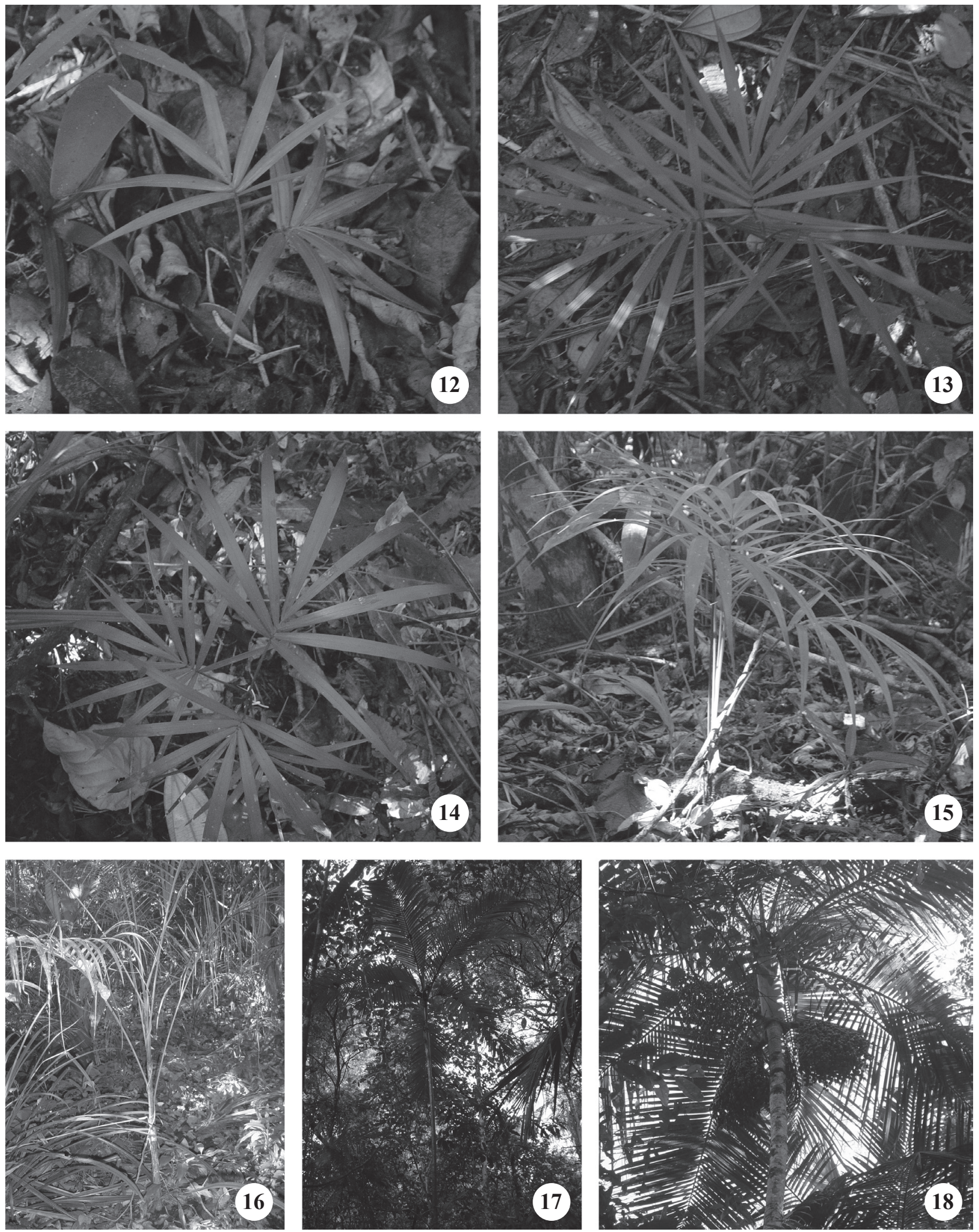

Figuras 12-18. Estádios ontogenéticos de Euterpe edulis nas áreas de estudo. 12-13. Plântula. 14-15. Infante. 16. Jovem. 17. Imaturo. 18. Reprodutivo. Mediana da altura dos indivíduos reprodutivos $=700 \mathrm{~cm}$.

Figures 12-18. Ontogenetic stages of Euterpe edulis in the studied areas. 12-13. Seedling. 14-15. Infant. 16. Juvenile. 17. Immature. 18. Reproductive. Hight median of reproductive adults $=700 \mathrm{~cm}$. 

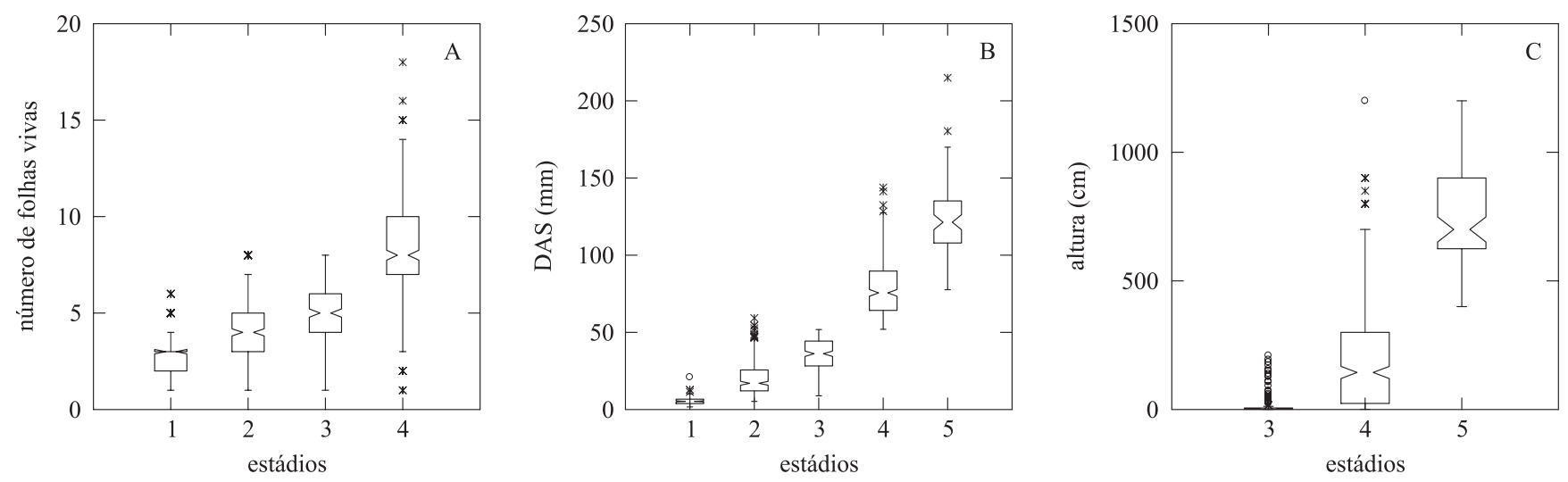

Figura 19. Número de folhas (A), DAS (B) e altura (C) de Euterpe edulis dos estádios ontogenéticos no Norte do Estado do Rio de Janeiro. 1. Plântula $(n=188) ; 2$. Infante $(n=292)$; 3. Jovem $(n=214)$; 4. Imaturo $(n=336)$; . Reprodutivo $(n=79)$. Cada caixa (box) representa $50 \%$ das observações, as barras verticais representam a amplitude total de distribuição; * = valores extremos (outlier); $\mathrm{O}=$ valores muito extremos. A porção "acinturada" da caixa representa o intervalo de confiança de $95 \%$ ao redor da mediana. Porções "acinturadas" que não se sobrepõem indicam diferenças significativas no nível de $95 \%$ de confiança.

Figure 19. Number of leaves (A), DAS (B) and height (C) of Euterpe edulis ontogenetic stages in the north of Rio de Janeiro State. 1. Seedling $(n=188) ; 2$. Infant $(n=292) ; 3$. Juvenile $(n=214) ; 4$. Immature $(n=336) ; 5$. Reproductive $(n=79)$. Each box represents $50 \%$ of the observation, the bars represent the amplitude of the observation; notches surrounding the median represent median $95 \%$ confidence intervals; $*=$ outlier; $\mathrm{O}=$ extreme value. Non-overlapping of notches indicates significant difference at $95 \%$ confidence level.

com DAS máximo de $30 \mathrm{~mm}$; imaturo ou virgem (figura 24) - indivíduo com presença de estipe aéreo com DAS maior que $30 \mathrm{~mm}$ mas sem sinal de evento reprodutivo; reprodutivo ou adulto (figura 25) - indivíduo com estipe aparente e com sinal de evento reprodutivo (presença de inflorescência ou infrutescência).

Plantas em diferentes estádios de G. schottiana apresentaram aumento no número de folhas com o desenvolvimento $(H=1624,291, g l=4, P<0,001)$ (figura 27), exceto nos estádios infante e jovem que apresentaram o mesmo número de folhas. O DAS $(H=885,318, g l=3, P<0,001)$ e a altura $(H=379,029$, $g l=2, P<0,001$ ) (figura 27) aumentaram com o desenvolvimento. O estádio imaturo não diferiu do reprodutivo apenas pela ausência de estrutura reprodutiva, mas também por apresentar tamanho diferente.

Geonoma schottiana ocorreu como indivíduos solitários e touceira. Porém, do total de 2.469 indivíduos amostrados em 2005 nos cinco fragmentos, apenas 4,9\% se apresentaram como touceira (tabela 4). O número de touceiras encontradas foi de 31 , com a mediana do número de indivíduos sendo 3 (mínimo de 2 indivíduos e máximo de 10). Não foi encontrada diferença do tamanho das touceiras entre os fragmentos $(H=1,83$, $g l=3, P=0,608)$. Nenhum indivíduo reprodutivo foi encontrado formando touceira.
Tabela 4. Número de indivíduos amostrados de Geonoma schottiana e de indivíduos em touceiras, seguindo pela porcentagem, na coleta de dados de 2005 nos cinco fragmentos de Mata Atlântica localizados no Norte do Estado do Rio de Janeiro. Área amostrada por fragmento: 0,81 ha. (Fragmento 1. Reserva Biológica Poço das Antas, 3.500 ha; Fragmento 2. Reserva Biológica União, 2.400 ha; Fragmento 3. Santa Helena, 57 ha; Fragmento 4. Estreito, 21 ha; Fragmento 5. Afetiva-Jorge, 19 ha).

Tabel 4. Single-stemmed and multi-stemmed number of individuals of all ontogenetic stages of Geonoma schottiana in 2005 in five forest fragments in the Atlantic Rain Forest in the north of Rio de Janeiro State. Total censused area in each fragment: 0.81 ha. (Fragment 1. Reserva Biológica Poço das Antas, 3,500 ha; Fragment 2. Reserva Biológica União, 2,400 ha; Fragment 3. Santa Helena, 57 ha; Fragment 4. Estreito, 21 ha; Fragment 5. Afetiva-Jorge, 19 ha).

\begin{tabular}{cccc}
\hline Fragmento & $\begin{array}{c}\text { Número de touceiras } \\
\text { amostradas }\end{array}$ & $\begin{array}{c}\text { Total de } \\
\text { indivíduos }\end{array}$ & $\begin{array}{c}\text { Indivíduos } \\
\text { em touceira }\end{array}$ \\
\hline 1 & 6 & 1827 & $18(0,98 \%)$ \\
2 & 0 & 6 & 0 \\
3 & 10 & 209 & $49(23,4 \%)$ \\
4 & 12 & 372 & $41(11,02 \%)$ \\
5 & 3 & 55 & $13(23,63 \%)$ \\
\hline Total & 31 & 2469 & $121(4,9 \%)$ \\
\hline
\end{tabular}



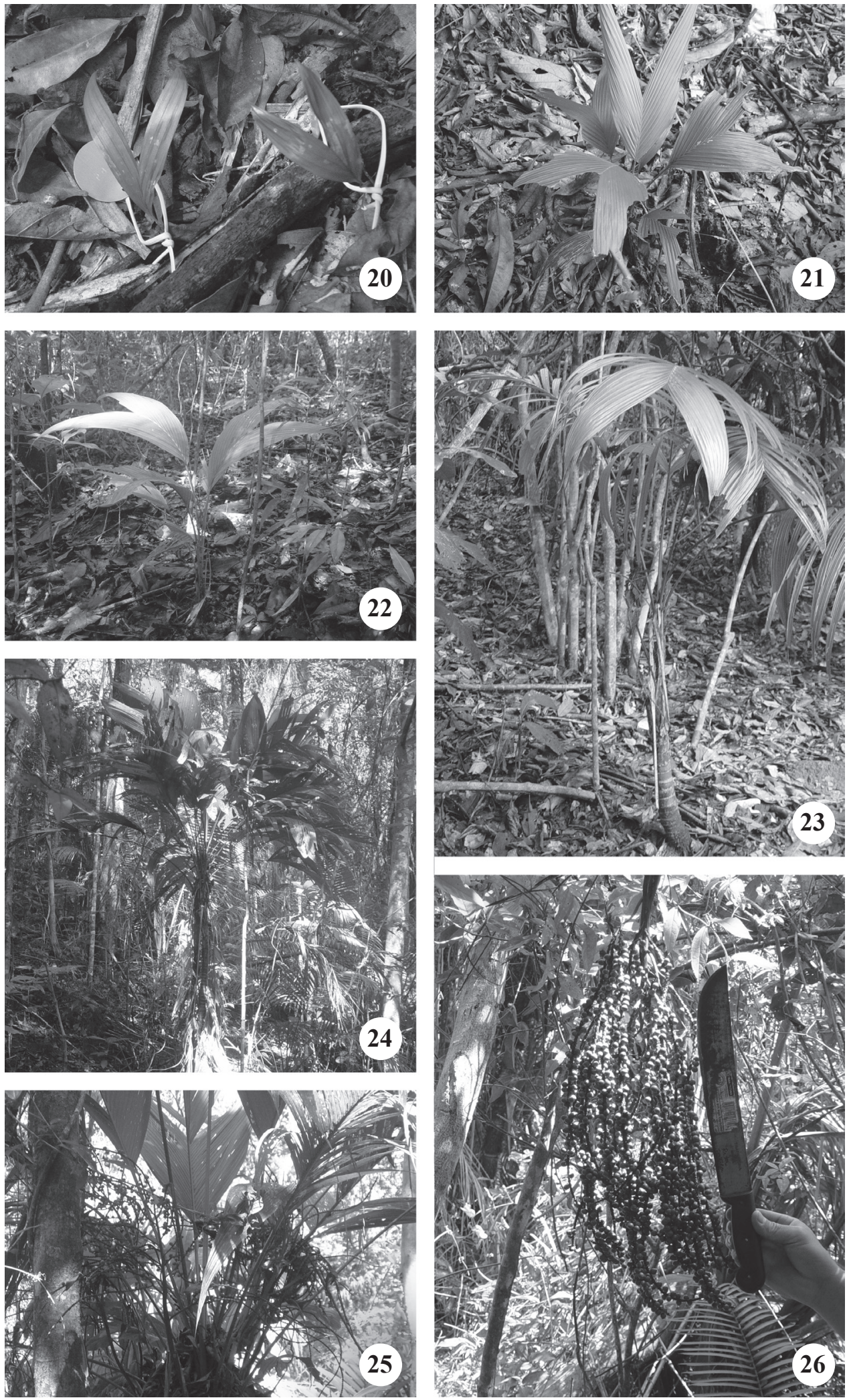

Figuras 20-26. Estádios ontogenéticos de Geonoma schottiana nas áreas de estudo. 20. Plântula. 21-22. Infante. 23. Jovem. 24. Imaturo. 25. Reprodutivo. 26. Frutos. Mediana da altura dos indivíduos reprodutivos $=124 \mathrm{~cm}$.

Figures 20-26. Ontogenetic stages of Geonoma schottiana in the studied areas. 20. Seedling. 21-22. Infant. 23. Juvenile. 24. Immature. 25. Reproductive. 26. Fruits. Hight median of reproductive adults $=124 \mathrm{~cm}$. 

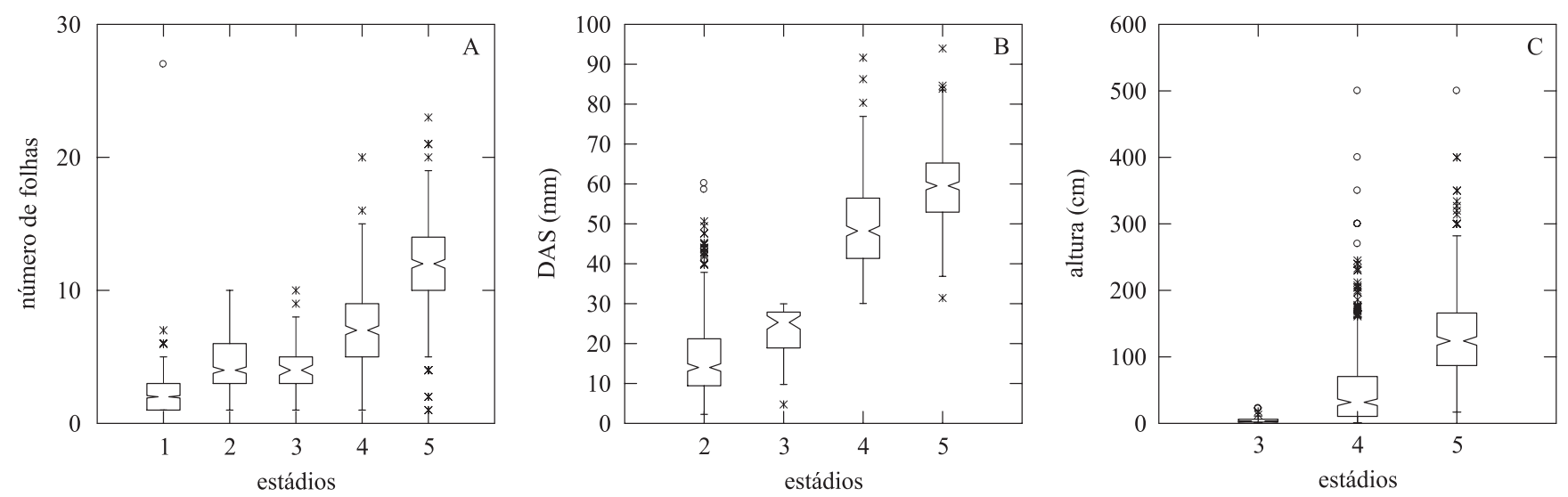

Figura 27. Número de folhas (A), DAS (B) e altura (C) de Geonoma schottiana dos estádios ontogenéticos no Norte do Estado do Rio de Janeiro. 1. Plântula $(n=1.261) ; 2$. Infante $(n=394) ; 3$. Jovem $(n=71) ; 4$. Imaturo $(n=368) ; 5$. Reprodutivo $(n=376)$. Cada caixa (box) representa 50\% das observações, as barras verticais representam a amplitude total de distribuição; * = valores extremos (outlier); $O$ = valores muito extremos. A porção "acinturada" da caixa representa o intervalo de confiança de $95 \%$ ao redor da mediana. Porções "acinturadas" que não se sobrepõem indicam diferenças significativas no nível de $95 \%$ de confiança.

Figure 27. Number of leaves (A), DAS (B) and height (C) of Geonoma schottiana ontogenetic stages in the north of Rio de Janeiro State. 1. Seedling $(n=1.261) ; 2$. Infant $(n=394) ; 3$. Juvenile $(n=71) ; 4$. Immature $(n=368) ; 5$. Reproductive $(n=376)$. Each box represents $50 \%$ of the observation, the bars represent the amplitude of the observation; notches surrounding the median represent median $95 \%$ confidence intervals; $*=$ outlier; $O=$ extreme value. Non-overlapping of notches indicates significant difference at $95 \%$ confidence level.

\section{Discussão}

As três espécies apresentaram diferenças marcantes nas suas características qualitativas ao longo da ontogenia. Tipo de folha, aparecimento do estipe e eventos reprodutivos foram as principais características usadas para identificação dos estágios ontogenéticos. Porém, para Euterpe edulis e Geonoma schottiana características quantitativas também foram utilizadas, pois alguns estádios apresentaram ampla variação no tamanho.

Os estádios ontogenéticos definidos para Astrocaryum aculeatissimum foram semelhantes aos estádios definidos para A. mexicanum (Piñero et al. 1984). Ambas as espécies possuem cinco estádios ontogenéticos, definidos por características qualitativas, tais como a perda de algumas características (folhas bífidas) e aparecimento de outras (folhas pinadas, estipe e estruturas reprodutivas).

Reis et al. (1996), Silva-Matos (1995) e Carvalho et al. (1999) apresentaram diferentes definições de estádios ontogenéticos/classes de tamanho para Euterpe edulis. Reis et al. (1996) usaram a altura para a definição de seis classes de tamanho, Silva-Matos (1995) definiu quatro classes de tamanho levando em consideração o número de folhas, DAS e altura, enquanto Carvalho et al. (1999) estudaram apenas os estádios pré-reprodutivos e definiram três classes de tamanho de acordo com o número de folhas, formato das folhas, altura e DAS. Nesse último estudo os estádios definidos foram plântulas: indivíduos com até três folhas leques, infantes: indivíduos já com a quarta folha, sendo estas com formato intermediário entre leque e pinada e jovens: indivíduos com folhas pinadas, altura mínima de $17 \mathrm{~cm}$ e DAS máximo de $52 \mathrm{~mm}$. Aqui, foram definidos quatro estádios e um dos estádios foi dividido em duas classes de tamanho. Foi seguida a recomendação de Gatsuk et. al. (1980) para a definição dos quatro estádios, onde o estádio de um indivíduo deve ser definido por características quantitativas e qualitativas e geralmente são caracterizadas pelo aparecimento de estruturas que estavam ausentes em estádios mais recentes de desenvolvimento e pela perda de outras que estavam presentes. Porém, para um dos estádios foi necessária a divisão em duas classes de tamanho, já que esse estádio abrange indivíduos de tamanhos muito diferentes e, portanto, podem apresentar características de mortalidade e natalidade dos indivíduos também muito diferentes. Os quatro estádios e a divisão de um em duas classes de tamanho definidos aqui levaram em conta a presença 
de estruturas embrionárias; plântulas com folhas leques; o aparecimento de uma estrutura madura; infantes com as primeiras folhas pinadas; o aparecimento do estipe; jovens e imaturos (um estádio e duas classes de tamanho), sendo separados por uma característica quantitativa - DAS; e finalmente os reprodutivos, pelo aparecimento de estruturas reprodutivas.

Geonoma schottiana apresentou na área de estudo a morfologia das folhas muito variável. Não foi encontrado um padrão de desenvolvimento na forma da folha ao longo do desenvolvimento da ontogenia da espécie, portanto esta característica qualitativa não ajudou na definição dos seus estádios ontogenéticos. Já em um estudo realizado por Sampaio \& Scariot (2010), com a mesma espécie em uma floresta de galeria em Brasília, os autores identificaram cinco estádios pós-germinativos: plântulas, que seriam indivíduos com folhas bífidas; infantes, com pelo menos uma folha iniciando a divisão; juvenil, já com pelo menos uma folha completamente pinada; imaturo, com estipe aparente e sem sinal de evento reprodutivo e reprodutivo. Os estádios identificados para a espécie no presente estudo e os identificados por Sampaio \& Scariot (2010) são bastante diferentes. A espécie na Mata Atlântica apresenta folhas de tipos muito variáveis, sendo essa característica insuficiente para a divisão dos estádios. Já no Cerrado, parece existir um padrão de desenvolvimento foliar mais definido. Essa diferença entre os resultados dos dois estudos pode estar relacionada com pressões ambientais diferentes entre os biomas estudados ou pode estar indicando a alta plasticidade fenotípica dos indivíduos ao longo de sua distribuição. Chazdon (1991) estudando 23 espécies do gênero Geonoma (G. schottiana não foi estudada), também encontrou grande variação na morfologia das folhas. As plântulas do gênero Geonoma sempre apresentam folhas bífidas, e durante a ontogenia muitas espécies apresentam uma transição gradual de folhas bífidas para folhas parcialmente ou totalmente divididas (Chazdon 1991). Outras espécies apresentam folhas bífidas por todo ciclo de vida. As principais características utilizadas para a definição de estádios ontogenéticos, assim como para G. brevispatha (Souza et al. 2003), foi ausência ou presença de estipe aparente e estruturas reprodutivas. Para essa espécie foram definidos três estádios ontogenéticos, sendo que dois deles foram divididos em 2 classes de tamanho, de acordo com o DAS.

Foi encontrado um aumento no número de folhas, do DAS e da altura (comprimento do estipe) com o desenvolvimento dos estádios ontogenéticos para as três espécies estudadas, confirmando assim resultados já relatados na literatura para outras espécies de palmeiras (Chazdon 1991, Carvalho et al. 1999, Souza et al. 2000, 2003), exceto para os infantes e jovens de G. schottiana que apresentaram o mesmo número de folhas. Nesse caso, como na transição de infantes para jovens ocorre o aparecimento do estipe, esse desenvolvimento pode levar a um deslocamento dos recursos de produção de folhas para o crescimento do estipe, e então não ocorrer o aumento de número de folhas como o esperado. Outra hipótese é que a dinâmica de folhas se estabilizou, não sendo possível um acúmulo maior de folhas, dada a longevidade delas e a taxa de produção. Com essa estratégia de crescimento, os dois estádios não apresentam diferenças em relação ao número de folhas, mas sim em relação ao DAS. Souza et al. (2003) trabalhando com Geonoma brevispatha encontraram que indivíduos menores investem mais no crescimento vertical do que na produção de folhas e que, possivelmente, essa estratégia está relacionada com a procura por maior luminosidade. O mesmo pode estar acontecendo com G. schottiana.

A presença de touceiras com maior número de indivíduos de Astrocaryum aculeatissimum nos menores fragmentos pode estar ligada a um maior investimento na reprodução assexuada em áreas onde a espécie tenha dificuldade no estabelecimento de suas plântulas (reprodução sexuada) (Portela 2008). Nas mesmas áreas deste estudo, Pires (2006) encontrou maior remoção de frutos nos maiores fragmentos. Portanto, outra explicação possível para esse padrão é a menor remoção de sementes nos menores fragmentos que leva as sementes a germinarem na própria touceira aumentando assim o tamanho destas. Em um estudo realizado com $A$. murumuru no Peru, comparando a remoção de sementes em touceiras com indivíduos solitários, ocorreu maior sobrevivência das sementes nas touceiras e maior número de sementes removidas nas proximidades de indivíduos solitários (Beck \& Terborgh 2002). Essa pode ser uma explicação para a maior parte da população de $A$. aculeatissimum (56,6\%) se apresentar como touceira.

Estudos que busquem a identificação e descrição de estádios ontogenéticos são muito importantes para possibilitar a padronização e comparação entre estudos populacionais. A partir de estudos como este, poderemos tirar conclusões mais precisas acerca das condições de populações em seus ambientes naturais e também comparar com mais segurança o comportamento de populações sob diferentes pressões ambientais e antrópicas. 
Agradecimentos - A Eraldo dos Santos Almeida e Antonio Tavares de Oliveira, pela ajuda na coleta de dados; à Fapesp (Proc. № 2005/60788-4), pela ajuda financeira e à Capes, pela bolsa de doutorado de R.C.Q.; ao CNPq pelo apoio financeiro através de Portela. F.A.M. Santos (Proc. № 304937/2007$0)$. Agradecemos, ainda, ao ICMBio e aos proprietários dos fragmentos pela permissão do desenvolvimento da pesquisa.

\section{Referências}

ALVES, L.F., MARTINS, F.R. \& SANTOS, F.A.M. 2004. Allometry of a neotropical palm, Euterpe edulis Mart. Acta Botanica Brasilica 18:369-374.

BECK, H. \& TERBORGH, J. 2002. Groves versus isolates: how spatial aggregation of Astrocaryum murumuru palms affects seed removal. Journal of Tropical Ecology 18:275-288.

BERNACCI, L.C., MARTINS, F.R. \& SANTOS, F.A.M. 2008. Estrutura de estádios ontogenéticos em população nativa da palmeira Syagrus romanzoffiana (Cham.) Glassman (Arecaceae). Acta Botanica Brasilica 22: 119-130.

CARVALHO, R.M., MARTINS, F.R. \& SANTOS, F.A.M. 1999. Leaf ecology of pre-reproductive ontogenetic stages of the palm tree Euterpe edulis Mart. (Arecaceae). Annals of Botany 83:225-233.

CHAZDON, R.L. 1991. Plant size and form in the understory palm genus Geonoma: are species variations on a theme? American Journal of Botany 78:680-694.

CHAZDON, R.L. 1996. Spatial heterogeneity in tropical forest structure: canopy palms as landscape mosaics. Trends in Ecology and Evolution 11:8-9.

GALETTI, M. \& ALEIXO, A. 1998. Effects of palm heart harvesting on avian frugivores in the Atlantic rain forest of Brazil. Journal of Applied Ecology 35:286-293.

GATSUK, L.E., SMIRNOVA, O.V., VORONTZOVA, L.V., ZAUGOLNOVA, L.B. \& ZHUKOVA, L.A. 1980. Age states of plants of various growth forms: a review. Journal of Ecology 68:675-696.

HENDERSON, A. 2011. A revision of Geonoma (Arecaceae). Phytotaxa 17:1-271.

HENDERSON, A., GALEANO, G. \& BERNAL, R., 1995. Field guide to the palms of the Americas. Princeton University Press, Princeton.
LORENZI, H., SOUZA, H.M., MEDEIROS-COSTA, J.T., CERQUEIRA, L.S.C. \& FERREIRA, E. 2004. Palmeiras brasileiras e exóticas cultivadas. Plantarum, Nova Odessa.

PIÑERO, D., MARTINEZ-RAMOS, M. \& SARUKHÁN, J. 1984. A population model of Astrocaryum mexicanum and a sensitivity analysis of its finite rate of increase. Jornal of Ecology 72:977-991.

PIRES, A.S. 2006. Perda da diversidade de palmeiras em fragmentos de Mata Atlântica: padrões e processos. Tese de doutorado, Universidade Estadual Paulista, Rio Claro.

PORTELA, R.C.Q. 2008. Dinâmica populacional de três espécies de palmeiras em uma paisagem fragmentada no domínio da Mata Atlântica, RJ. Tese de doutorado, Universidade Estadual de Campinas, Campinas.

PORTELA, R.C.Q., PIRES, A.S. \& SANTOS, F.A.M. 2009. Unusual branching in the palm Euterpe edulis Mart. Hoehnea 36:501-506.

REIS, A., KAGEYAMA, P., REIS, M.S. \& FANTINI, A.C. 1996. Demografia de Euterpe edulis (Arecaceae) em uma Floreta Ombrófila Densa Montana em Blumenau (SC). Sellowia 47:3-33.

SCARIOT, A. 1996. The effects of rain forest fragmentation on the palm community in Central Amazonia. Tese de doutorado, University of California, Santa Barbara.

SAMPAIO, M.B. \& SCARIOT, A. 2010. Effects of stochastic herbivory events on population maintenance of an understorey palm species (Geonoma schottiana) in riparian tropical forest. Journal of Tropical Ecology 26:151-161.

SILVA-MATOS, D.M. 1995. Population ecology of Euterpe edulis Mart. (Palmae). Tese de doutorado, University of East Anglia, Norwich.

SOUZA, A.F., MARTINS, F.R. \& SILVA-MATOS, D.M. 2000. Detecting ontogenetic stages of the palm Attalea humilis in fragments of the Brazilian Atlantic forest. Canadian Journal of Botany 78:1227-1237.

SOUZA, A.F., MARTINS, F.R. \& BERNACCI, L.C. 2003. Clonal growth and reproductive strategies of the understory tropical palm Geonoma brevispatha: an ontogenetic approach. Canadian Journal of Botany 81:101-112.

SYSTAT. 2004. Standard version, version 11. SPSS Inc., Chicago, Illinois. 